\title{
Optimised Low-Thrust Mission to the Atira Asteroids
}

\author{
Marilena Di Carlo ${ }^{1}$ \\ Department of Mechanical and Aerospace Engineering, University of Strathclyde, 75 \\ Montrose Street, G1 1XJ Glasgow, United Kingdom \\ Juan Manuel Romero Martin \\ Department of Mechanical and Aerospace Engineering, University of Strathclyde, 75 \\ Montrose Street, G1 1 XJ Glasgow, United Kingdom \\ Natalia Ortiz Gomez \\ Engineering and the Environment, University of Southampton, University Road SO17 \\ 1BJ, United Kingdom \\ Massimiliano Vasile \\ Department of Mechanical and Aerospace Engineering, University of Strathclyde, 75 \\ Montrose Street, G1 1XJ Glasgow, United Kingdom
}

\begin{abstract}
Atira asteroids are recently-discovered celestial bodies characterised by orbits lying completely inside the heliocentric orbit of the Earth. The study of these objects is difficult due to the limitations of ground-based observations: objects can only be detected when the Sun is not in the field of view of the telescope. However, many asteroids are expected to exist in the inner region of the Solar System, many of which could pose a significant threat to our planet. In this paper, a small, low-cost, mission to visit the known Atira asteroids and to discover new Near Earth Asteroids (NEA) is proposed. The mission is realised using electric propulsion. The trajectory is optimised
\end{abstract}

\footnotetext{
Email addresses: marilena.di-carlo@strath.ac.uk (Marilena Di Carlo), juan.romero-martin@strath.ac.uk (Juan Manuel Romero Martin), n.ortiz-gomez@soton.ac.uk (Natalia Ortiz Gomez), massimiliano.vasile@strath.ac.uk (Massimiliano Vasile)

${ }^{1}$ Corresponding author
} 
to maximise the number of visited asteroids of the Atira group using the minimum propellant consumption. During the tour of the Atira asteroids an opportunistic NEA discovery campaign is proposed to increase our knowledge of the asteroid population. The mission ends with a transfer to an orbit with perihelion equal to Venus's orbit radius. This orbit represents a vantage point to monitor and detect asteroids in the inner part of the Solar System and provide early warning in the case of a potential impact.

Keywords: Atira asteroids; low-thrust; trajectory optimisation

\section{Introduction}

Atira asteroids are Near-Earth Asteroids (NEAs) with both perihelion and aphelion within the orbit of the Earth (aphelion $Q<0.983 \mathrm{AU}$ ), also called Inner-Earth Objects (IEOs). The first Atira object was discovered in 2003 and, as of March 2016, sixteen asteroids are counted in this group (see Table $1)^{2}$. However, many more objects are expected to exist in the same region of the Solar System. To date, over eleven thousand NEAs have been identified, the majority of which are characterized by semimajor axis greater than $1 \mathrm{AU}$, as shown in Figure 1, where the distribution of the known NEAs is shown in the $a-e$ and $a-i$ planes, with the Atira asteroids represented by triangular markers.
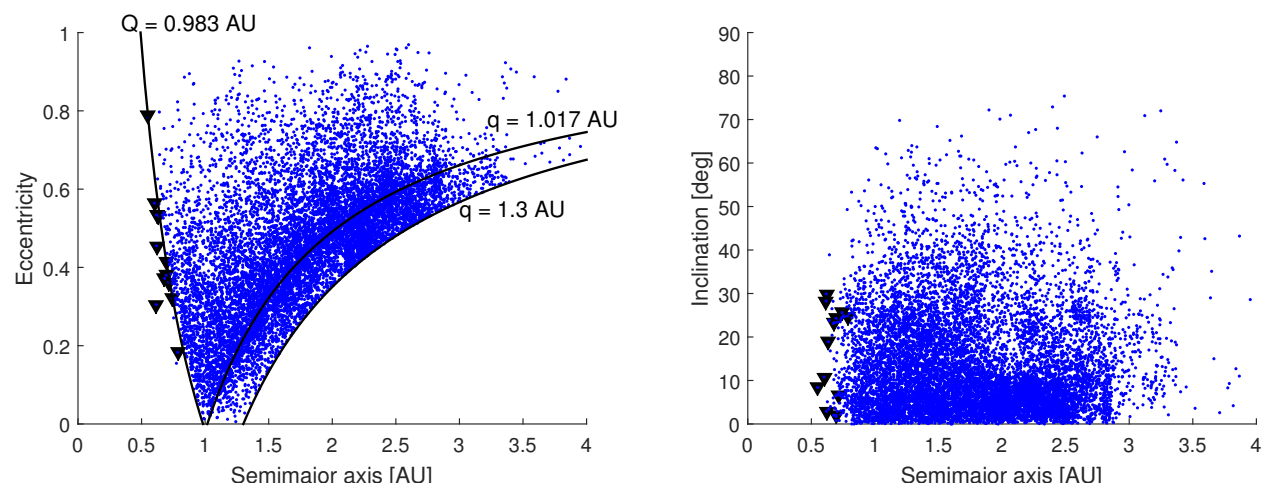

Figure 1: NEAs distribution in the $a-e$ and $a-i$ planes; red circles indicate Atira asteroids.

\footnotetext{
${ }^{2}$ JPL Small-Body Database Search Engine - http://ssd.jpl .nasa.gov/sbdb_query . $\operatorname{cgi}$
} 
Table 1: Orbital elements of the known Atira asteroids: semi-major axis (a), eccentricity $(e)$, inclination $(i)$, longitude of the ascending node $(\Omega)$, argument of perihelion $(\omega)$, mean anomaly $(M)$ and time of passage at perihelion $\left(t_{p}\right)$. The asteroids shown in bold are the ones known when the study started and the ones considered in this paper.

\begin{tabular}{llllllll}
\hline Object ID & $a$ & $e$ & $i$ & $\Omega$ & $\omega$ & $M$ & $t_{p}$ \\
& {$[\mathrm{AU}]$} & & {$[\mathrm{deg}]$} & {$[\mathrm{deg}]$} & {$[\mathrm{deg}]$} & {$[\mathrm{deg}]$} & {$[\mathrm{ET}]$} \\
\hline 1998 DK36 & 0.69 & 0.42 & 2.02 & 151.46 & 180.04 & 183.25 & 1998-Jun-07.3 \\
2003 CP20 & 0.74 & 0.32 & 25.62 & 103.92 & 252.93 & 336.80 & 2014 -Dec-24.0 \\
2004 XZ130 & 0.62 & 0.45 & 2.95 & 211.41 & 5.16 & 23.25 & 2014 -Nov-27.6 \\
2004 JG6 & 0.64 & 0.53 & 18.94 & 37.04 & 352.98 & 168.72 & 2014 -Sep-13.3 \\
2005 TG45 & 0.68 & 0.37 & 23.33 & 273.46 & 230.42 & 324.97 & 2014 -Dec-29.0 \\
2006 WE4 & 0.78 & 0.18 & 24.77 & 311.04 & 318.62 & 44.70 & 2014 -Nov-07.5 \\
2007 EB26 & 0.55 & 0.79 & 8.49 & 63.22 & 236.71 & 237.91 & $2007-$ Apr-30.2 \\
2008 EA32 & 0.62 & 0.30 & 28.27 & 100.97 & 181.85 & 216.53 & 2015-Feb-17.4 \\
2008 UL90 & 0.69 & 0.38 & 24.31 & 81.17 & 183.61 & 358.46 & 2014-Dec-09.9 \\
2010 XB11 & 0.62 & 0.53 & 29.88 & 96.32 & 202.48 & 223.70 & 2015 -Feb-14.2 \\
2012 VE46 & 0.71 & 0.36 & 6.67 & 8.95 & 190.36 & 8.52 & 2014-Dec-03.8 \\
2013 JX28 & 0.60 & 0.56 & 10.76 & 39.97 & 354.88 & 311.78 & 2014-Dec-31.8 \\
2013 TQ5 & 0.77 & 0.16 & 16.38 & 286.77 & 247.32 & 83.30 & 2014 -Oct-12.5 \\
2014 FO47 & 0.75 & 0.27 & 19.18 & 358.68 & 347.41 & 225.84 & 2015-Mar-07.8 \\
2015 DR215 & 0.67 & 0.47 & 4.10 & 315.05 & 42.17 & 48.53 & 2015-Dec-17.2 \\
2015 ME131 & 0.80 & 0.19 & 28.88 & 314.36 & 164.03 & 189.74 & 2015-Oct-26.7 \\
\hline
\end{tabular}

Inner Solar System asteroids are indeed difficult to discover and track because Earth-bound telescopes have difficulties detecting asteroids when the Sun is in the field of view. For this reason these asteroids could represent a hazard for our planet. The object that exploded in an air burst over Chelyabinsk, in Russia, in February 2013, injuring more than 1,000 people, approached, undetected, from the Sun direction.

In recent years, successful missions such as Deep Space 1 (Rayman \& Williams (2002)), Hayabusa (Kawaguchi (2011)) and Dawn (Brophy et al. (2008)) have demonstrated the possibility to successfully survey or even land on asteroids in our Solar System. However, to date no mission has targeted inner Solar System asteroids, including members of the Atira group. The aforementioned missions made use of electric propulsion systems to achieve shorter flight time, smaller launch vehicles and increased mass delivered to destination, when compared to high-thrust propulsion systems (Williams \& 
Coverstone-Carroll (1997); Sauer \& Yen (1995)).

This paper proposes a mission to visit the known Atira asteroids, using a spacecraft equipped with an electric propulsion system. In order to maximise the scientific return of the mission, the trajectory is optimised to visit the maximum possible number of asteroids of the Atira group while conducting a discovery campaign to detect new Near-Earth Asteroids.

The paper presents the most important aspects of the analysis and design of the mission, including the launch and orbit injection strategy. This analysis will demonstrate that a tour of the Atira is achievable with a small spacecraft and a low-cost launcher. Furthermore, it will be demonstrated that the same spacecraft can be injected in a heliocentric surveillance orbit that serves as vantage point to observe, detect and monitor asteroids in the inner Solar System.

The paper is structured as follows. Section 2 presents an overview of the mission design process. The methods used to find the sequence of visited asteroids and optimise the associated low-thrust trajectory are described in Sections 3 and 4, including different strategies to achieve the final surveillance orbit. The study of the launch and orbit injection strategy is presented in Section 5; the study of the visibility analysis of inner-Earth asteroids is described in Section 6 and the results obtained are presented in Section 7.

\section{Mission Design Overview}

The encounters with the Atira asteroids are realised through a series of fly-by's at the nodal points of the orbits of the asteroids. The mission design process is divided into three phases:

1. Sequence Finder: identification of the optimal sequence of asteroids to visit, departure and arrival dates using an impulsive Lambert model for the transfers (Section 3).

2. Additional Optimisation: refinement of the optimal solution found at the previous step using a Differential Evolution-based single objective global optimisation algorithm (Section 3.1).

3. Low-Thrust Trajectory Optimisation: translation of the optimal impulsive solution found at the previous step into a low-thrust optimal trajectory (Section 4).

After the last fly-by the spacecraft is injected into a reduced perihelion surveillance orbit using the electric propulsion system (Section 4). The base- 
line trajectory solution is then used to analyse the feasibility of a low-cost launch opportunity (Section 5).

Given that the main objective is to maximise the number of visited asteroids with the minimum propellant mass, the trajectory is not optimised for the discovery campaign. On the contrary a visibility analysis is carried out in order to assess the number of asteroids that could potentially be observed along the baseline trajectory derived from point 3 here above (Section 6).

\section{Sequence Finder}

The definition of the optimal sequence of asteroids and departure and arrival dates at the nodes of the asteroids, requires the solution of a combinatorial optimisation problem. This problem is solved using an algorithm called "Lambert problem to Target Asteroids at Nodal points" (LambTAN).

LambTAN is inspired to the general branch-and-prune techniques and in particular the incremental pruning techniques proposed by Becerra et al. (2003) and Novak \& Vasile (2010). Complete trajectories are incrementally constructed by adding one transfer arc at a time following a tree structure in which each branch is a possible partial sequence of asteroids. Partial transfers are pruned out if one or more criteria are not met (for example, the $\Delta V$ of an arc is greater than a given maximum value).

The asteroids are assumed to move on Keplerian orbits as a solution of a two-body Sun-Asteroid problem. The trajectories are composed of sequences of conic arcs linked together through discrete, instantaneous events. Each conic arc is the solution of a Lambert problem, which is solved to compute the $\Delta V$ required for the spacecraft to hop from one asteroid to another. Asteroids are met at their nodal points to avoid expensive inclination change manoeuvres. At every hop the spacecraft is transferred to a new heliocentric orbit. For the following hop a new set of departure conditions are identified along the current heliocentric orbit, within a minimum and maximum value for the time of flight to reach the nodal point of the next asteroid. As an example, Figure 2 shows several Lambert arcs reaching a given asteroids from different departure points on the Earth's orbit.

The search space is pruned in order to exclude non-feasible solutions for the low-thrust optimisation as well as considering constraints for the maximum local departure $\Delta V$, the minimum and maximum time of flights and the minimum perihelion. 


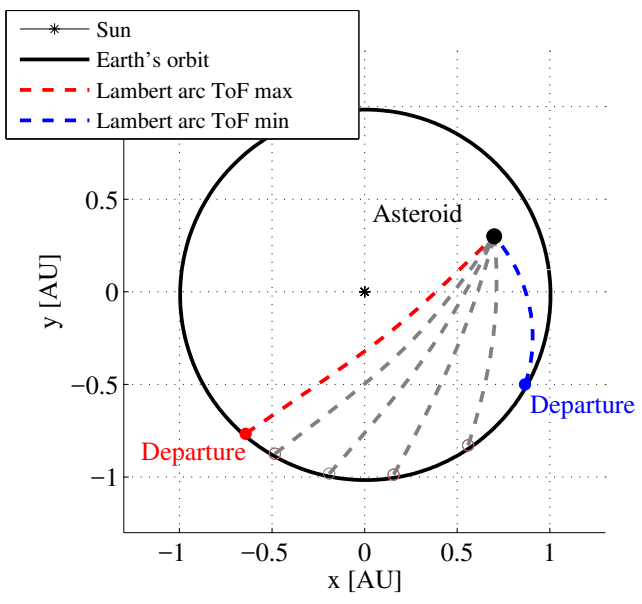

Figure 2: Lambert arcs for Earth-to-asteroid transfer example. The asteroid fly-by occurs at the nodal point. Subsequent asteroid-to-asteroid transfers are computed in analogous fashion.

The algorithm starts, for a transfer $j$, from a given departure orbit $\mathfrak{T}_{j}$, identified by its orbital elements:

$$
\mathfrak{T}_{j}=\{a, e, i, \Omega, \omega\},
$$

where $a$ is the semimajor axis, $e$ is the eccentricity, $i$ is the inclination, $\Omega$ is the right ascension of the ascending node and $\omega$ is the argument of the perihelion.

For a given set of target asteroids $S_{j}=\left\{A_{1}, A_{2}, \ldots, A_{d}\right\}$, the ascending and descending nodal points are computed. For the $k$-th asteroid, $A_{k}$, the epochs of passage through the ascending and descending nodal points, $T_{A_{k}}^{a s c}$ and $T_{A_{k}}^{\text {desc }}$, are computed within the interval of time going from the considered epoch, $T$, to the end of the mission, $T_{\text {end }}$. For each node passing epoch $T_{A_{k}}^{a s c}$ and $T_{A_{k}}^{\text {desc }}$, a window for the departure times from the departure orbit $\mathfrak{T}_{j}$ can be computed. In particular, the start $T_{\text {start }}^{D W}$ and end $T_{\text {end }}^{D W}$ epochs for the departure window $D W$ are computed by subtracting the minimum and maximum time of flight $T o F_{\min }$ and $T o F_{\max }$ from each node passing epoch $T_{A_{k}}^{a s c}$ and $T_{A_{k}}^{\text {desc }}$. For an encounter at the ascending node at time $T_{A_{k}}^{a s c}$ :

$$
\begin{aligned}
T_{\text {start }}^{D W} & =\max \left[\left(T_{A_{k}}^{a s c}-T o F_{\max }\right), T_{A_{k-1}}\right] \\
T_{\text {end }}^{D W} & =T_{A_{k}}^{a s c}-T_{o} F_{\text {min }} .
\end{aligned}
$$


where $T_{A_{k-1}}$ is the passing time at the previous visited asteroid. In Eq. (2), $T_{A_{k}}^{a s c}$ has to satisfy $T_{A_{k}}^{a s c} \leq T_{\text {end }}$ where $T_{\text {end }}$ is the mission end epoch. The Lambert arc associated with departure time $T_{\text {start }}^{D W}$ is represented in red in Figure 2 while the Lambert arc associated with departure time $T_{\text {end }}^{D W}$ is shown in blue. The next step is to compute, from the window of departure times going from $T_{\text {start }}^{D W}$ to $T_{\text {end }}^{D W}, n$ Lambert arcs that connect the departure orbit $\mathfrak{T}_{j}$ with the nodal point at the considered nodal passing epoch, as shown in Figure 2. The value $n$ is given by:

$$
n=\frac{T_{\text {end }}^{D W}-T_{\text {start }}^{D W}}{T_{o} F_{\text {step }}},
$$

where ToF step is the considered step size for the time of flights. As an example, in Figure 2, ToF $F_{\text {step }}$ is such as to give $n=6$.

For each Lambert arc, the algorithm proceeds to the next step only if three constraint criteria are met:

1. the $\Delta V$ at departure for the current Lambert arc, $\Delta V_{a r c_{k}}$, does not exceed a given maximum value, $\Delta V_{\max }$ :

$$
\Delta V_{a r c_{k}} \leq \Delta V_{\text {max }}
$$

2. the Lambert transfer is characterised by a perihelion $q$ greater than a given minimum perihelion, $q_{\min }$ :

$$
q>q_{\text {min }}
$$

3. the impulsive Lambert transfer can be realised with the low-thrust propulsion system. This is deemed possible if the following condition is satisfied:

$$
\operatorname{ToF}_{a r c_{k}} \epsilon \geq \max \left[C \Delta V_{a r c_{k}}, \sqrt{V_{0}^{2}-2 V_{f} V_{0}+V_{f}^{2}}\right]
$$

where $T o F_{a r c_{k}}$ is the time of flight for the current Lambert arc, $\Delta V_{a r c_{k}}$ is the change in velocity required for the impulsive Lambert arc at departure, $\epsilon$ is the acceleration provided by the low-thrust engine, $C$ is an appropriate empirical coefficient, $V_{0}$ is the spacecraft's velocity when it passes the previous asteroid and $V_{f}$ is the velocity at the end of the Lambert arc. The second term in square brackets in the previous equation is the Edelbaum's $\Delta V$ for low-thrust transfer between circular orbits (Chobotov (2002)). 
Table 2: LambTAN parameters settings.

\begin{tabular}{ll}
\hline$S$ & Target asteroids $\left[A_{1}, A_{2}, \ldots A_{d}\right]$ \\
$T_{0}$ & Mission start epoch \\
$T_{\text {end }}$ & Mission end epoch \\
$T_{o} F_{\max }$ & Maximum Time of Flight for each Lambert arc \\
$T_{o} F_{\min }$ & Minimum Time of Flight for each Lambert arc \\
$T o F_{\text {step }}$ & Time step for the Time of Flight \\
$\Delta V_{\max }$ & Maximum departure velocity vector \\
& {$\left[\Delta V_{\text {Earth }}, \Delta V_{\text {arc } 1}, \Delta V_{\text {arc } 2}, \ldots, \Delta V_{\text {arcn }}\right]$} \\
$q_{\min }$ & Minimum perihelion \\
$\epsilon$ & Low-thrust acceleration \\
$C$ & Scaling factor \\
\hline
\end{tabular}

If the considered Lambert arc meets all of the above constraints, then the Lambert arc is set as the new departure orbit for the next transfer, $\mathfrak{T}_{j+1}$. A new set of possible target asteroids, $S_{j+1}$, is defined by removing the asteroid visited at step $j, A_{j}$, from the set of possible targets, $S_{j+1}=\left\{x: x \in S_{j} \wedge x \neq\right.$ $\left.A_{j}\right\}$. The process is then repeated for all possible combinations of asteroids.

A full solution is generated when the set of target asteroids $S$ is empty, $S=$ $\emptyset$, or if the end time of the mission epoch has been reached. Representing with $N_{A}$ the number of visited asteroids, the complete solution vector provided by LambTAN is:

$$
\mathbf{x}_{\text {LambTAN }}=\left[T_{1}, T_{o} F_{1}, A_{1}, T_{2}, T_{o} F_{2}, A_{2}, \ldots, T_{N_{A}}, T_{o} F_{N_{A}}, A_{N_{A}}\right]^{T},
$$

where, for the first transfer, $T_{1}$ is the departure time, $T_{o} F_{1}$ is the time of flight for the Lambert arc and $A_{1}$ is the target asteroid. The main setting parameters of the LambTAN solver are summarized in Table 2.

\subsection{Additional Optimisation}

The optimisation analysis previously described results in many solutions, each one characterised by a specific set of variables (number of asteroid visited, asteroids sequence, departure dates, time of flights). The solutions obtained are ranked in order to identify the ones with maximum sequence length and lowest $\Delta V$. The first ranked solutions is then further optimised by means of an evolutionary single objective global optimisation algorithm called AIDEA (Adaptive Inflationary Differential Evolution Algorithm) (Minisci \& 
Vasile (2014)). AIDEA is an adaptive stochastic optimiser which combines Differential Evolution (DE), (Price et al. (2006)), with the working principles of Monotonic Basin Hopping Algorithm (MBH) (Wales \& Doye (1997)). For more details on AIDEA please refer to Minisci \& Vasile (2014) and related work by the authors.

The optimisation executed using AIDEA is realised in order to improve the departure dates found by LambTAN, leading to a reduced $\Delta V$ for the transfers. In order to do so, a time window of $\pm \xi$ days is allocated around each departure date $T_{i}$ identified by LambTAN and AIDEA is run to find, in this search space, departure dates leading to a reduced value of the total $\Delta V$. The values of the times of flight are derived from the requirement to encounter the asteroids at one of their nodes and, thus, are not optimised further. Considering the solution vector provided by LambTAN, see Eq. (7), the lower and upper boundaries, LB and UB of the search space for AIDEA are defined as:

$$
\begin{gathered}
\mathbf{L B}=\left[T_{1}-\xi, T_{2}-\xi, \ldots, T_{N_{A}}-\xi\right]^{T}, \\
\mathbf{U B}=\left[T_{1}+\xi, T_{2}+\xi, \ldots, T_{N_{A}}+\xi\right]^{T} .
\end{gathered}
$$

\section{Low-Thrust Trajectory Optimisation}

The outcome of the sequence finder and optimisation with AIDEA is a sequence of transfer arcs characterised by a departure state vector, an end state vector, a transfer time and a departure $\Delta V$. The low-thrust optimisation process determines, for each transfer arc, an optimal control history, for the low-thrust engine of the spacecraft, to depart from the initial state vector and achieve the desired end position and velocity in the given transfer time.

In this study we use a variant of the direct analytical multiple shooting algorithm proposed by Zuiani et al. (Zuiani et al. (2012)) and implemented in the software code FABLE (FAst Boundary-value Low-thrust Estimator). The transfer arc is split into a predefined sequence of $n_{L T}$ finite coast and thrust legs. Each $s$-th leg is represented by a vector of equinoctial parameters $\mathbf{E}_{s}=\left[a_{s}, P_{1, s}, P_{2, s}, Q_{1, s}, Q_{2, s}, L_{s}\right]^{T}$, plus, in case of thrust arc, the low-thrust acceleration components, $a_{r}, a_{t}$ and $a_{h}$ expressed in a local radial-transversal 
reference frame, (Zuiani et al. (2012)), as:

$$
\mathbf{a}_{L T, s}=\left\{\begin{array}{l}
a_{r} \\
a_{t} \\
a_{h}
\end{array}\right\}=\left\{\begin{array}{c}
\epsilon_{i} \cos \alpha_{i} \cos \beta_{i} \\
\epsilon_{i} \sin \alpha_{i} \cos \beta_{i} \\
\epsilon_{i} \sin \beta_{i}
\end{array}\right\}
$$

where $\alpha_{s}, \beta_{s}$ and $\epsilon_{s}$ are, respectively, the azimuth, elevation and modulus of the acceleration and $\epsilon_{s}=F_{s} / m_{s}$ is simply the ratio between thrust $F_{s}$ and mass of the spacecraft $m_{s}$.

The trajectory is then analytically propagated forward from the departure point and backward from the end point (Figure 3). The motion is assumed purely Keplerian along coast legs while thrust legs are analytically propagated using the asymptotic expansion solutions proposed in (Zuiani \& Vasile (2015)). Each leg begins and ends at an On/Off control node, where On nodes define the switching point from a coast to a thrust leg and Off nodes define the switching point from a thrust to a coast leg (see Figure 3). Therefore, thrust legs are defined by a set of orbital elements at an On node, $E_{s}^{O N}$, and coast legs are defined by a set of orbital elements at an Off node, $E_{s}^{O F F}$ (see Figure 3).

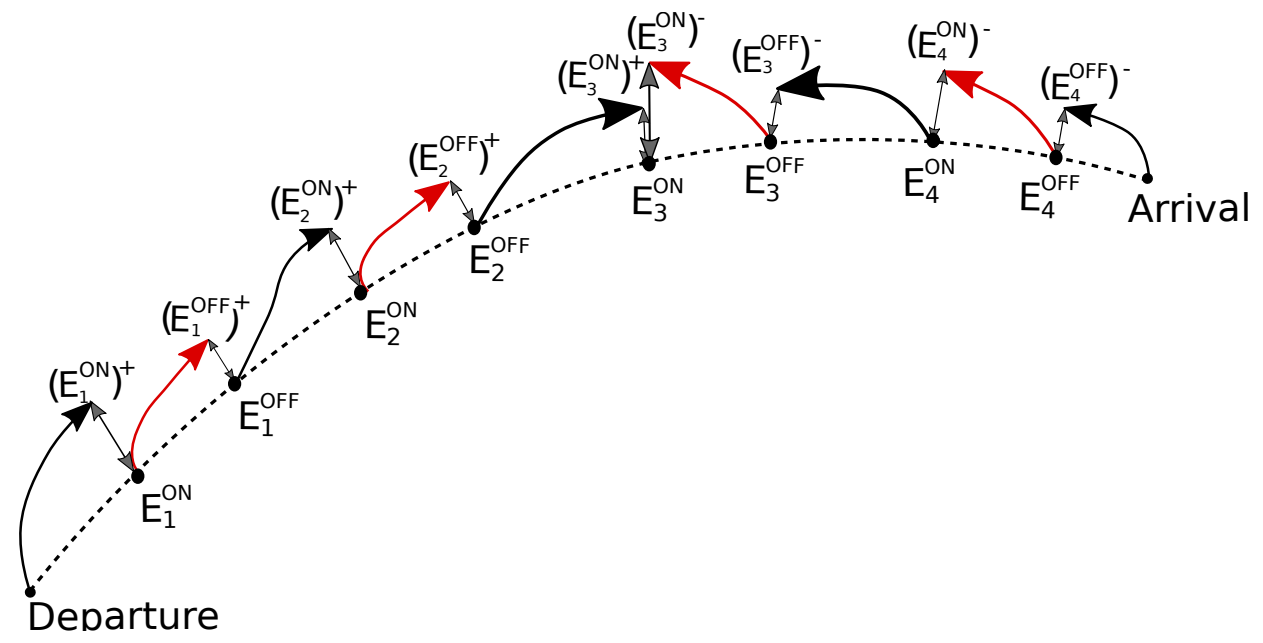

Figure 3: Segmentation of the trajectory into coast legs (black) and thrust legs (red).

For the trajectories considered in this study, the angle $\beta$ is set to zero, since the transfers are all on the ecliptic plane. The azimuth angles $\alpha_{s}$ are instead optimisation variables while the modulus $\epsilon$ of the acceleration depends only 
on the mass of the spacecraft. The mass of the spacecraft is conservatively kept constant over each transfer arc and updated at the end of the transfer according to the propellant mass spent to realise that transfer.

The optimisable vector for each transfer is, therefore, defined by the azimuth angles $\alpha_{s}$, for each thrust arc, and the equinoctial elements at each On and Off point:

$$
\mathbf{x}_{L T}=\left[\alpha_{1}, \mathbf{E}_{1}^{O N}, \mathbf{E}_{1}^{O F F}, \alpha_{2}, \mathbf{E}_{2}^{O N}, \mathbf{E}_{2}^{O F F}, \alpha_{n_{L T}}, \mathbf{E}_{n_{L T}}^{O N}, \mathbf{E}_{n_{L T}}^{O F F}\right]^{T}
$$

where $n_{L T}$ is the number of thrust and coast legs. The optimisation problem is formulated as a non-linear programming problem whose objective is the total $\Delta V$ for each transfer

$$
\min _{\mathbf{x}_{L T}} \Delta V=\sum_{s} \epsilon_{s} \Delta t_{s}\left(\mathbf{x}_{L T}\right)
$$

where $\Delta t_{s}\left(\mathbf{x}_{L T}\right)$ is the time length of each thrust leg, subject to the following constraints:

$$
\begin{cases}\left(\mathbf{E}_{1}^{O N}\right)^{+}=\mathbf{E}_{1}^{O N} & \\ \left(\mathbf{E}_{s}^{O F F}\right)^{+}=\mathbf{E}_{s}^{O F F} & s=1, \ldots, n_{L T} / 2 \\ \left(\mathbf{E}_{s}^{O N}\right)^{-}=\mathbf{E}_{s}^{O N} & s=n_{L T} / 2+1, \ldots, n_{L T} \\ \left(\mathbf{E}_{n L T / 2+1}^{O N}\right)^{+}=\left(\mathbf{E}_{n L T / 2+1}^{O N}\right)^{-} & \\ \left(\mathbf{E}_{n L T}^{O F F}\right)^{-}=\mathbf{E}_{n L T}^{O F F} & \\ \sum_{s=1}^{n_{L T}} \Delta t_{s}=T o F & \end{cases}
$$

The plus and minus signs in the constraints equations (Eq. (13)) indicate, respectively, the forward integration leg and the backward integration leg.

In order to facilitate convergence to a solution of the non-linear programming problem defined in Eqs. (12) and (13), a continuation method (Bonnard eg al. (2005)) over the modulus of the thrust is implemented. The non-linear programming problem is solved using the Matlabß fmincon-sqp algorithm.

\subsection{Transfer to the Surveillance Orbit}

After the fly-by with the last asteroid in the sequence identified by LambTAN, the spacecraft is moved to an orbit with reduced perihelion, equal to $0.725 \mathrm{AU}$, in order to continue its observation of NEAs. Three strategies were 
considered for this transfer: 1) a low-thrust spiral, 2) a low-thrust transfer exploiting resonances with the Earth, 3) a low-thrust transfer exploiting one Earth's gravity assist. The design of these possibilities is briefly described in the following paragraphs.

1. Low-thrust spiral. The optimisation problem is analogous to the one previously described but without the constraint on the transfer time in Eqs. (13).

2. Low-thrust and Earth's Resonances. The spiral is timed to take advantage of the gravity perturbation of the Earth to change the perihelion of the orbit. The Earth can perturb the orbit of the spacecraft when the Earth is in the vicinity of the apohelion of the orbit of the spacecraft at the same time that the spacecraft is at the apohelion (Stock (2009)). In particular, if the Earth is ahead of the spacecraft the perihelion will be increased, while if the Earth is behind the spacecraft the perihelion will be decreased. For this study a reduction of perihelion is sought.

3. Low-thrust and Earth's swing-by. The low-thrust engine is used to inject the spacecraft, after the last fly-by, into a trajectory that encounters the Earth, so that a gravity assist that reduces the perihelion can be realised. The gravity assist is modeled with the linked conic approximation proposed in Vasile \& Pascale (2006).

The trajectory optimisation problem is modified including the time of the swing-by of the Earth, $T_{G A}$, and the radius of the perigee of the hyperbola at the Earth, $r_{p}^{G A}$. The new solution vector becomes:

$\mathbf{x}_{L T}^{G A}=\left[\alpha_{1}, \mathbf{E}_{1}^{O N}, \mathbf{E}_{1}^{O F F}, \alpha_{2}, \mathbf{E}_{2}^{O N}, \mathbf{E}_{2}^{O F F}, \ldots, \alpha_{n_{L T}}, \mathbf{E}_{n_{L T}}^{O N}, \mathbf{E}_{n_{L T}}^{O F F}, T_{G A}, r_{p}^{G A}\right]^{T}$

Since the arrival state vector is not known a priori, the backward propagation is not implemented in this case. The non-linear programming problem is solved minimising the propellant consumption subject to constraints (13), only for the forward propagation leg, plus the following two additional constraints:

- The orbit of the spacecraft after the gravity assist with the Earth has perihelion equal to $0.725 \mathrm{AU}$;

- The encounter with the Earth takes place after a coast arc with minimum duration of 30 days to minimise the risks during critical phases of the mission. 


\section{Launch and Orbit Injection}

The mission proposed in this paper aims at achieving the desired scientific objectives with a small spacecraft and a low-cost launch opportunity. In order to meet this expectation the launch and orbit injection strategy needs to be carefully considered. Two options were analysed; injecting the spacecraft into an escape trajectory with the right velocity at the Earth to reach the first asteroid with no low-thrust manoeuvres or injecting the spacecraft into an escape trajectory that provides zero relative velocity with respect to the Earth and then using the low-thrust engine to reach the first asteroid. The former strategy provided the best results in terms of total $\Delta V$, and launch cost and will be described in the following.

The assumption is that the launcher places the spacecraft on a Geostationary Transfer Orbit (GTO), with orbital elements $a_{G T O}, e_{G T O}, i_{G T O}$ and $\omega_{G T O}$, and provides an re-ignitable upper stage. The heliocentric velocity of the spacecraft on its departure from Earth, $\mathbf{v}_{\text {initial }}$, is obtained from the solution provided by the LambTAN algorithm. The relative velocity vector with respect to Earth at the departure is simply $\mathbf{v}_{\infty}=\mathbf{v}_{\text {initial }}-\mathbf{v}_{\oplus}$, where $\mathbf{v}_{\oplus}$ is the velocity of the Earth expressed in the heliocentric reference frame. The vector $\mathbf{v}_{\infty}$ is then transformed from the heliocentric to the planetocentric reference frame in order to obtain its declination $\delta$, required to compute the inclination $i$ of the hyperbolic orbit, according to (Sorensen (2003)):

$$
\sin i=\frac{\sin \delta}{\sin \left(\omega_{G T O}+\theta\right)},
$$

where $\theta$ is the true anomaly corresponding to the asymptotic direction (Kemble (2006)). The angle $\theta$ can be computed using the following equations:

$$
\begin{gathered}
a_{\text {hyp }}=-\frac{\mu_{\oplus}}{\left\|\mathbf{v}_{\infty}\right\|^{2}} \\
e_{\text {hyp }}=1-\left(\frac{a_{G T O}\left(1-e_{G T O}\right)}{a_{\text {hyp }}}\right)
\end{gathered}
$$

and

$$
\theta=\arccos \left(-\frac{1}{e_{\text {hyp }}}\right)
$$

The computed values of $\delta$ and $\theta$ could be such that using them together with $\omega_{G T O}$ in Eq. (15) would give $\sin i>1$. In this case, since $\delta$ is defined by 
the geometry of the initial velocity, a change of $i$ and $\omega$ is required to insert the spacecraft into the appropriate hyperbolic orbit. It is therefore assumed that the upper stage can provide multiple manoeuvres to change $i$ and $\omega$.

In the following we will consider a two manoeuvre injection strategy:

1. the first manoeuvre, executed at the ascending node of the GTO orbit, change the inclination from $i_{G T O}$ to $i_{i n j}=i_{G T O}+\Delta i$;

2. the second manoeuvre is executed at an appropriate position along the GTO orbit with inclination $i_{i n j}$ so that the injection into the hyperbolic orbit, with an appropriate value of $\omega_{i n j}$, can be obtained.

The values of $i_{i n j}$ and $\omega_{i n j}$ corresponding to the lowest $\Delta V$ for the two manoeuvres are computed using the following procedure:

- the true anomaly of the point where the second manoeuvre takes place is $\left(2 \pi-\omega_{G T O}+\omega_{i n j}\right)$ (Figure 4$)$. Using this value of the true anomaly, the position and velocity of the spacecraft at the point along the orbit where the hyperbolic injection manoeuvre is executed are computed, for each value of $\omega_{i n j} \in[0,2 \pi]$, using:

$$
r_{i n j}=\frac{a_{G T O}\left(1-e_{G T O}^{2}\right)}{1+e_{G T O} \cos \left(2 \pi-\omega_{G T O}+\omega_{i n j}\right)}
$$

and

$$
v_{i n j}=\sqrt{\frac{\mu_{\oplus}}{a_{G T O}\left(1-e_{G T O}^{2}\right)}} \sqrt{1+e_{G T O}^{2}+2 e_{G T O} \cos \left(2 \pi-\omega_{G T O}+\omega_{i n j}\right)}
$$

- the eccentricity of the hyperbola is computed, for each value of $\omega_{i n j} \in$ $[0,2 \pi]$, using:

$$
e_{h y p}=1-\frac{r_{i n j}}{a_{h y p}}
$$

with $a_{\text {hyp }}$ from Eq. (16);

- values of $\theta$ are computed using Eq. (18);

- the inclination $i_{i n j}$ is computed using $\delta$ from Eq. (15) and $\theta$ resulting from the previous step for every value of $\omega_{i n j} \in[0,2 \pi]$; 


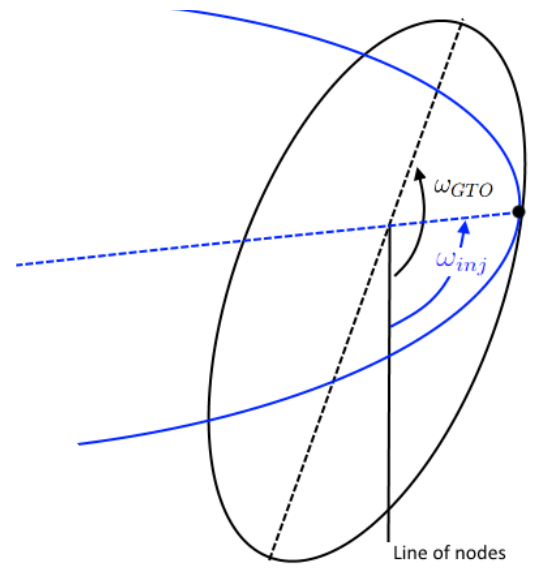

Figure 4: Orbit geometry for the second manuever: the GTO with inclination $i_{i n j}$ is in black, the hyperbolic orbit is in blue.

- the $\Delta V$ required to perform the variation of inclination with the first manoeuvre is computed as:

$$
\Delta V_{i}=2 V_{a s c} \sin \left(\frac{\Delta i}{2}\right)
$$

where $\Delta i=i_{i n j}-i_{G T O}$ and $V_{a s c}$ is the velocity at the ascending node of the orbit:

$$
V_{a s c}=\sqrt{\frac{\mu_{\oplus}}{a_{G T O}\left(1-e_{G T O}^{2}\right)}} \sqrt{1+e_{G T O}^{2}+2 e_{G T O} \cos \left(2 \pi-\omega_{G T O}\right)}
$$

- the variation of velocity required to perform the second manoeuvre and inject the spacecraft into the hyperbolic orbit is computed as:

$$
\Delta V_{i n j}=\sqrt{V_{h y p}^{2}+V_{i n j}^{2}-2 V_{h y p} V_{i n j} \cos \gamma_{i n j}}
$$

where $V_{\text {hyp }}$ is the velocity at the perigee on the hyperbolic orbit:

$$
V_{\text {hyp }}=\sqrt{\frac{2 \mu_{\oplus}}{r_{i n j}}+\left\|\mathbf{v}_{\infty}\right\|^{2}}
$$

and $\gamma_{i n j}$ is the flight path angle at the point of the manoeuvre:

$$
\tan \gamma_{i n j}=\frac{e_{G T O} \sin \left(2 \pi-\omega_{G T O}+\omega_{i n j}\right)}{1+e_{G T O} \cos \left(2 \pi-\omega_{G T O}+\omega_{i n j}\right)}
$$


- finally, the total velocity is:

$$
\Delta V_{\text {total }}=\Delta V_{i}+\Delta V_{i n j}
$$

The values of $i_{i n j}$ and $\omega_{i n j}$ leading to the lower value of $\Delta V_{\text {total }}$ are used to realise the two manoeuvres to inject the spacecraft into its hyperbolic orbit.

\section{Visibility Analysis}

During the transfer from one asteroid to another, observations of the inner part of the Solar System can be carried out with appropriate instrumentation placed on-board of the spacecraft, in order to detect new NEAs. The observations are carried out also during the transfer to and on the surveillance orbit. This analysis provides insight into the likelihood of observing new NEAs based on the current population.

The instrument that has been selected for this mission is the same one used for the Canadian microsatellite NEOSSat, launched in 2003. This instrument has a limiting relative magnitude $V$ of 19.5 with an exposure time of 100 seconds (Wallace et al. (2014)).

\subsection{Near Earth Asteroids Population}

In this study, the expected number and size of the asteroids potentially observable during the mission lifetime are derived from the available catalog of known NEAs. The database of known NEAs is taken from the JPL SmallBody Database Search Engine ${ }^{3}$.

Approximately 9,000 NEAs are currently known however, in this work we start from the working assumption that more than $10^{6}$ objects with absolute magnitude lower than $H=26$ exist. This assumption is in agreement with the NEA population estimate by Harris (Harris (2014)) and is used to evaluate the quality of the proposed survey. In line with the work of Greenstreet et al. (Greenstreet et al. (2012)) we introduce the further assumption that the existing population is composed of $30.1 \%$ of Amors asteroids, $63.3 \%$ of Apollo asteroids, $5 \%$ of Atens asteroids and 1.6\% of Inner Earth Objects.

With these two assumptions in mind, we generate a synthetic population by re-sampling the joint distribution of the orbital elements of the known

\footnotetext{
${ }^{3}$ JPL Small-Body Database Search Engine - http://ssd.jpl.nasa.gov/sbdb_query.cgi\#x
} 
NEAs. The existing population is then added to the synthetic one for consistency. The orbital elements of the synthetic and true populations are defined at a given epoch and the orbits of all the asteroids are then propagated forward in time for the duration of the mission. To generate the synthetic population, a total of 1,430,600 IEOs, Atens, Apollos and Amors are generated by re-sampling the existing joint distribution. Then, from the obtained synthetic population 22,890 IEOs, 71,531 Atens and 905,579 Apollos are selected, so that the sum is $10^{6}$ NEAs. The number of objects in each group respect the percentages given by Greenstreet.

The absolute magnitude $H$ of the asteroids of the synthetic populations is obtained considering the cumulative distribution (Stuart (2003))):

$$
N(<H)=10^{-3.88+0.39 H}
$$

The probability density function of the absolute magnitude $H, f_{H}$, is computed by normalising Equation (28) as follows:

$$
f_{H}=\frac{1}{C_{H}} \cdot 10^{-3.88+0.39 H}
$$

where $C_{H}$ is given by:

$$
C_{H}=\int_{H(N=1)}^{H\left(N=10^{6}\right)} 10^{-3.88+0.39 H} d H
$$

so that $f_{H}$ can be expressed as:

$$
f_{H}=8.9810^{-7} \cdot 10^{-3.88+0.39 H}
$$

The slope parameter $\mathrm{G}$ of the asteroids of the population has been modeled considering a distribution with mean value and standard deviation as proposed by Veres et al. (Veres et al. (2015)).

In order to obtain statistically significant results, 100 different synthetic populations of $10^{6}$ IEOs, Atens and Apollos asteroids are generated and an independent visibility analysis is run for each population.

\subsection{Observation Constraints}

In order for the on-board camera to detect an asteroid, the following three constraints have to be met:

- the asteroid has to be within the Field of View (FOV) of the camera; 


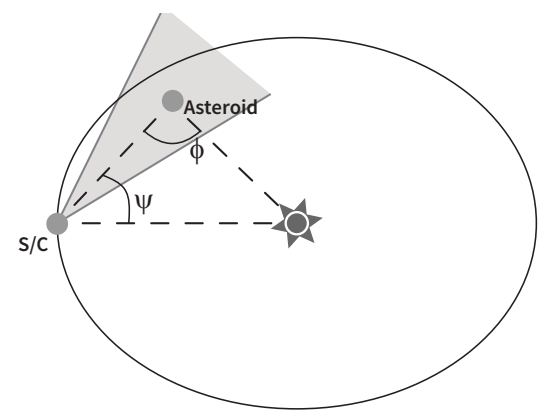

Figure 5: Camera pointing towards the inner part of the spacecraft trajectory.

- the asteroid relative magnitude $V$ with respect to the camera has to be below the camera detection threshold;

- the phase angle $\phi$ Sun-Asteroid-Spacecraft (Figure 5) has to be below a certain threshold.

The first two constraints are linked to the field of view of the camera and its limiting magnitude detection capability. The third constraint takes into account the necessary illumination conditions for the asteroid to be observed by the spacecraft. The best illumination conditions take place when $\phi=0$, that is when the spacecraft is between the Sun and the asteroid. The asteroid relative magnitude $V$ is given by (Stuart (2003)):

$$
V=5 \log _{10}(r \Delta)+H-2.5 \log _{10}\left((1-G) \lambda_{1}+G \lambda_{2}\right)
$$

where

$$
\lambda_{1}=\exp \left(-\left(3.33 \tan \left(\frac{\phi}{2}\right)\right)^{0.63}\right)
$$

and

$$
\lambda_{2}=\exp \left(-\left(1.87 \tan \left(\frac{\phi}{2}\right)\right)^{1.22}\right)
$$

In the previous equations $r$ is the distance between the asteroid and the Sun in AU, $\Delta$ is the distance between the asteroid and the spacecraft in AU, $\phi$ is the phase angle between the position vector asteroid-Sun and the position vector asteroid-spacecraft and $G$ is the slope parameter of the asteroid. 
The diameter of the NEAs is related to the absolute magnitude as shown in Eq. (35), (Tedesco (1994)),

$$
D=\frac{1329}{\sqrt{p_{v}}} 10^{-0.2 H},
$$

where $D$ is the diameter in $\mathrm{km}$ and $p_{v}$ is the albedo of the asteroid.

\section{Results}

In this section the results obtained using the methods described in the previous sections are presented.

\subsection{Sequence Finder}

The parameters used for the LambTAN solver are summarised in Table 1:

- the set of considered asteroids, $S$, comprises the first twelve asteroids from Table 1 (shown in bold in Table 1);

- the maximum mission time is 10 years, with initial epoch 01 January 2020 and final epoch 01 January 2030;

- the minimum and maximum times of flight for each transfer are 30 and 365 days, with steps of 10 days;

- the maximum departure $\Delta V$ for the Lambert transfer, $\Delta V_{\max }$, is 3 $\mathrm{km} / \mathrm{s}$ for departures from Earth and $1.5 \mathrm{~km} / \mathrm{s}$ for departures from other orbits;

- the minimum perihelion $q_{\text {min }}$ for the Lambert transfer is $0.31 \mathrm{AU}$;

- the considered low-thrust acceleration $\epsilon$ is $10^{-4} \mathrm{~m} / \mathrm{s}^{2}$ (corresponding to a $700 \mathrm{~kg}$ spacecraft with thrust equal to $0.07 \mathrm{~N}$, Section 7.3) and the parameter $C$ for the translation of the impulsive transfer into a low-thrust transfer is set to 2 . This value was obtained from a preliminary experimental test campaign where each transfer generated by LambTAN was re-optimised with the low-thrust solver.

LambTAN finds 133,761 solutions, with longest solutions characterised by six fly-bys; in Figure 6 the total $\Delta V$ required for all the solutions of four, five or six asteroids fly-by's are presented. 
Table 3: LambTAN simulation parameters.

\begin{tabular}{ll}
\hline$S$ & Asteroid in bold in Table 1 \\
$T_{0}$ & $01 / 01 / 2020$ \\
$T_{\text {end }}$ & $01 / 01 / 2030$ \\
$T o F_{\text {max }}$ & 365 days \\
$T o F_{\min }$ & 30 days \\
$T o F_{\text {step }}$ & 10 days \\
$\Delta V_{\text {MaxDep }}$ & $3 \mathrm{~km} / \mathrm{s}$ from Earth \\
& $1.5 \mathrm{~km} / \mathrm{s}$ from transfer orbits \\
$q_{\text {min }}$ & $0.31 \mathrm{AU}$ \\
$\epsilon$ & $10^{-4} \mathrm{~m} / \mathrm{s}^{2}$ \\
$C$ & 2 \\
\hline
\end{tabular}

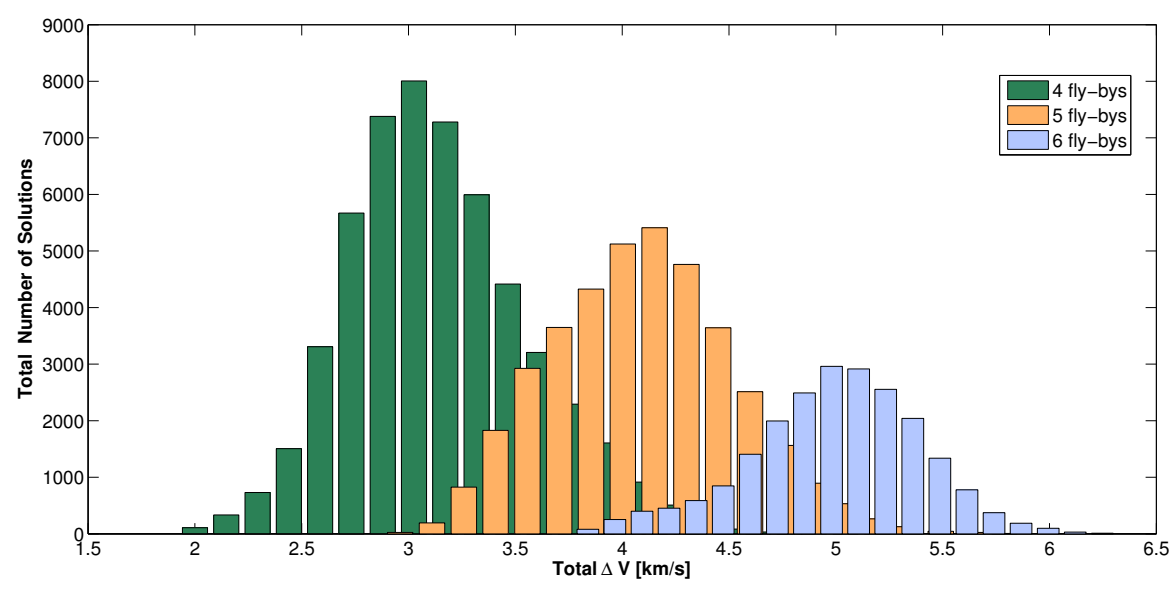

Figure 6: Solution distribution. 
Since many of the solutions are characterised by the same sequences of asteroids but different departure dates, a filtering process is applied to identify solutions targeting different asteroids. After the filtering, fourteen solutions with different sequence of asteroids, visiting six asteroids, and fifty-seven different solutions visiting five asteroids are found.

The best solution found by LambTAN, that is, the one characterised by the maximum number of asteroid visited and the lowest total $\Delta V$, has six fly-bys based on the following sequence: Earth - 2013 JX28 - 2006 WE4 2004 JG6 - 2012 VE46 - 2004 XZ130 - 2008 UL90 with a total $\Delta V$ cost of $3.77 \mathrm{~km} / \mathrm{s}$ and a transfer time of about 8.4 years, summarized in Table 4 .

Considering a typical chemical propulsion specific impulse of $I_{s p}=321 \mathrm{~s}$ and a spacecraft dry mass of approximately $595 \mathrm{~kg}$ at the end of the six fly-bys (as found in Table 6), realizing the six fly-by's with a chemical engine would require $1377.36 \mathrm{~kg}$ of propellant.

The positions of the targeted nodal points are shown in Figure 7 in the $x$ - $y$ plane of a heliocentric inertial reference frame.

Table 4: Best solution obtained with six visited asteroids using LambTAN.

\begin{tabular}{|c|c|c|c|c|c|c|}
\hline & Asteroid & $\begin{array}{l}\text { Departure } \\
\text { date }\end{array}$ & $\begin{array}{l}\text { ToF } \\
\text { [days] }\end{array}$ & $\begin{array}{l}\text { Arrival } \\
\text { date }\end{array}$ & $\begin{array}{l}\Delta V \\
{[\mathrm{~km} / \mathrm{s}]}\end{array}$ & $\begin{array}{l}m_{\text {fuel }} \\
{[\mathrm{kg}]}\end{array}$ \\
\hline & 2013 JX28 & $2020 / 09 / 29$ & 205 & $2021 / 04 / 22$ & 0.87 & 476.54 \\
\hline & 2006 WE4 & $2022 / 05 / 14$ & 215 & $2022 / 12 / 15$ & 0.86 & 357.79 \\
\hline & 2004 JG6 & $2023 / 06 / 14$ & 235 & $2024 / 02 / 04$ & 0.61 & 200.60 \\
\hline & 2012 VE46 & $2024 / 09 / 11$ & 265 & $2025 / 06 / 03$ & 0.36 & 101.37 \\
\hline & 2004 XZ130 & $2026 / 09 / 15$ & 205 & $2027 / 04 / 08$ & 0.73 & 173.15 \\
\hline & 2008 UL90 & $2028 / 07 / 31$ & 195 & $2029 / 02 / 11$ & 0.34 & 67.91 \\
\hline TOT. & & & & & 3.77 & 1377.36 \\
\hline
\end{tabular}

\subsection{Refinement of the Best Solution}

The best solution identified by LambTAN is further optimised using AIDEA (Section 3.1). For the additional optimisation a local window of $\xi=10$ days is allocated around the previous defined departure dates in order to identify new departures dates leading to an improved result in term of total $\Delta V$. The semi-amplitude of the local window is 10 days because of the value chosen for $T_{o} F_{\text {step }}$ in LambTAN. The maximum number of function evaluations for AIDEA is 2000. The settings of the parameters for AIDEA are $\delta_{\text {local }}=0.1$ 


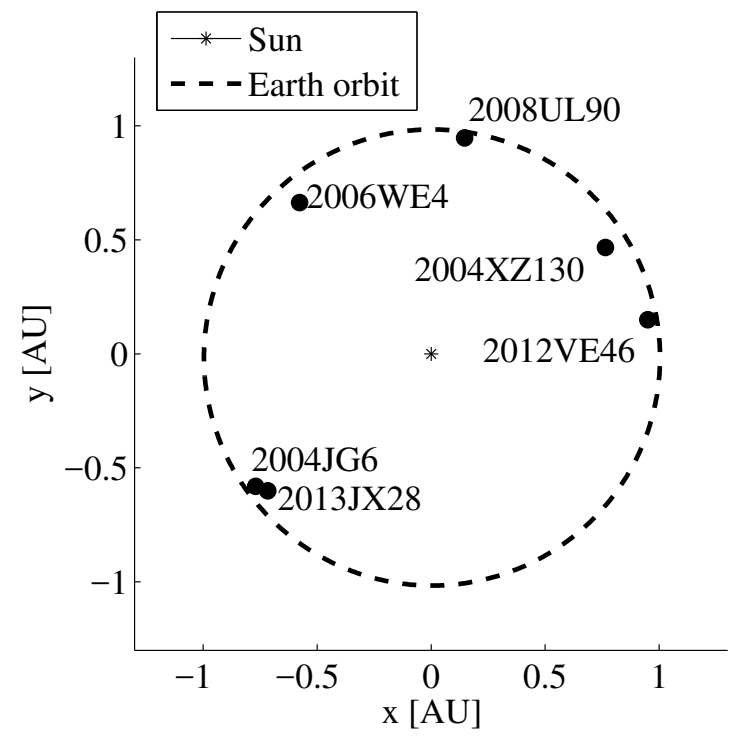

Figure 7: Targeted nodal points of the visited asteroids.

and $n_{L R}=10$ (Minisci \& Vasile (2014)). The obtained results are reported in Table 5 , showing a reduction of $0.16 \mathrm{~km} / \mathrm{s}$ in the total $\Delta V$ and of 103.76 $\mathrm{kg}$ in the fuel consumption with respect to the results presented in Table 4.

This solution and the results shown in Table 5 have been used for the optimisation of the low-thrust trajectory.

\subsection{Low-Thrust Trajectory Optimisation}

For the low-thrust optimisation the initial acceleration is set to $10^{-4} \mathrm{~m} / \mathrm{s}^{2}$, equivalent to a thrust $T=0.07 \mathrm{~N}$ applied to a $700 \mathrm{~kg}$ spacecraft. The specific impulse considered is $I_{s p}=3000 \mathrm{~s}$. The number of thrust legs for each transfer can vary between $n_{L T}=2$ and $n_{L T}=8$ and the initial acceleration for the continuation method varies between 1 and 25 times the nominal acceleration. The final solution for each transfer is the one obtained with the number of thrust arc $n_{L T}$ and the initial acceleration value for the continuation method that provide the lower $\Delta V$.

Following the strategy described in Section 7.4, the spacecraft is injected into an interplanetary orbit that meets the first asteroid without any thrust leg. After the first fly-by the engine is switched on to achieve the remaining five fly-by's. The resulting low-thrust trajectory is reported in Table 6 and 
Table 5: Further optimisation of the best solution obtained with six visited asteroids using AIDEA.

\begin{tabular}{|c|c|c|c|c|c|}
\hline Asteroid & $\begin{array}{l}\text { Departure } \\
\text { date }\end{array}$ & $\begin{array}{l}\text { ToF } \\
\text { [days] }\end{array}$ & $\begin{array}{l}\text { Arrival } \\
\text { date }\end{array}$ & $\begin{array}{l}\Delta V \\
{[\mathrm{~km} / \mathrm{s}]}\end{array}$ & $\begin{array}{l}m_{\text {fuel }} \\
{[\mathrm{kg}]}\end{array}$ \\
\hline 2013 JX28 & $2020 / 09 / 20$ & 214.5329 & $2021 / 04 / 22$ & 0.95 & 487.06 \\
\hline 2006 WE4 & $2022 / 05 / 24$ & 205 & $2022 / 12 / 15$ & 0.69 & 272.10 \\
\hline 2004 JG6 & $2023 / 06 / 12$ & 236.2514 & $2024 / 02 / 04$ & 0.61 & 195.56 \\
\hline 2012 VE46 & $2024 / 09 / 05$ & 270.6114 & $2025 / 06 / 03$ & 0.34 & 93.62 \\
\hline 2004 XZ130 & $2026 / 09 / 18$ & 201.5318 & $2027 / 04 / 08$ & 0.72 & 167.80 \\
\hline 2008 UL90 & $2028 / 08 / 10$ & 185.0003 & $2029 / 02 / 11$ & 0.29 & 57.46 \\
\hline & & & & 3.61 & 1273.60 \\
\hline
\end{tabular}

shown in Figure 8, where the thrust arcs are in black and the coast arcs are in gray.

Table 6: Summary of the simulation results for the low-thrust trajectory.

\begin{tabular}{llllll}
\hline Asteroid & $\begin{array}{l}\text { Time Engine } \\
\text { On [days] }\end{array}$ & $m_{0}[\mathrm{~kg}]$ & $m_{f}[\mathrm{~kg}]$ & $\Delta V[\mathrm{~km} / \mathrm{s}]$ \\
\hline 2013 JX28 & 0 & 700 & 700 & \\
2006 WE4 & 129.05 & 700 & 673.45 & 1.12 \\
2004 JG6 & 152.57 & 673.45 & 642.07 & 1.37 \\
2012 VE46 & 41.77 & 642.07 & 633.47 & 0.40 \\
2004 XZ130 & 158.40 & 633.47 & 600.89 & 1.51 \\
& 2008 UL90 & 30.04 & 600.89 & 594.17 & 0.30 \\
\hline TOTAL & & & & 4.70 \\
\hline
\end{tabular}

\subsubsection{Transfer to the Surveillance Orbit}

The transfer to the surveillance orbit using only a low-thrust spiral is reported in Table 7 and Figure 9. The total $\Delta V$ for this option is:

$$
\Delta V_{\text {fly-by }}+\Delta V_{\text {parking-orbit }}=(4.70+1.79) \mathrm{km} / \mathrm{s}=6.49 \mathrm{~km} / \mathrm{s} .
$$

In this case the low-thrust engine enables the entire mission to be achieved with approximately $145 \mathrm{~kg}$ of propellant, so that a spacecraft with a dry mass of $555 \mathrm{~kg}$ (Table 7) can be brought to the final surveillance orbit starting from a launch mass of $700 \mathrm{~kg}$. This is a remarkable improvement over the 


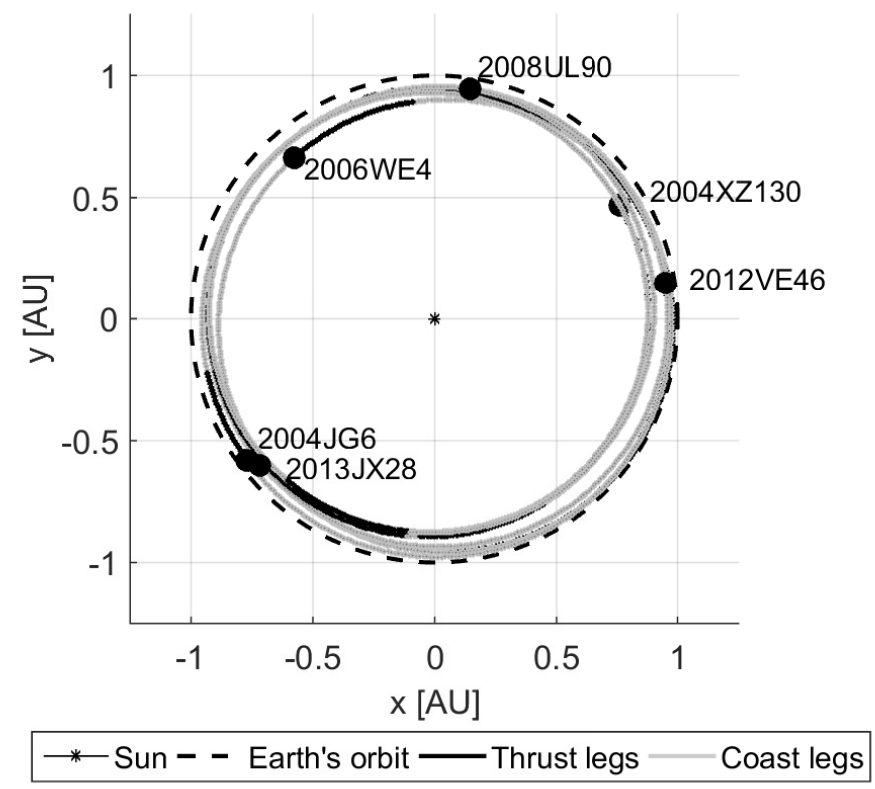

Figure 8: Low-thrust trajectory to visit six Atira asteroids at their nodal points. Coast legs are shown in gray and thrust legs in black.

Table 7: Summary of transfer to surveillance orbit after final fly-by.

\begin{tabular}{lllllll}
\hline Departure date & $\begin{array}{l}\text { ToF } \\
{[\text { days }]}\end{array}$ & $\begin{array}{l}\text { Engine on } \\
{[\text { days }]}\end{array}$ & $\begin{array}{l}\text { Arrival } \\
\text { date }\end{array}$ & $\begin{array}{l}m_{0} \\
{[\mathrm{~kg}]}\end{array}$ & $\begin{array}{l}m_{f} \\
{[\mathrm{~kg}]}\end{array}$ & $\begin{array}{l}\Delta V \\
{[\mathrm{~km} / \mathrm{s}]}\end{array}$ \\
\hline $2029 / 02 / 11$ & 421.78 & 176.83 & $2030 / 04 / 09$ & 594.17 & 557.80 & 1.79 \\
\hline
\end{tabular}




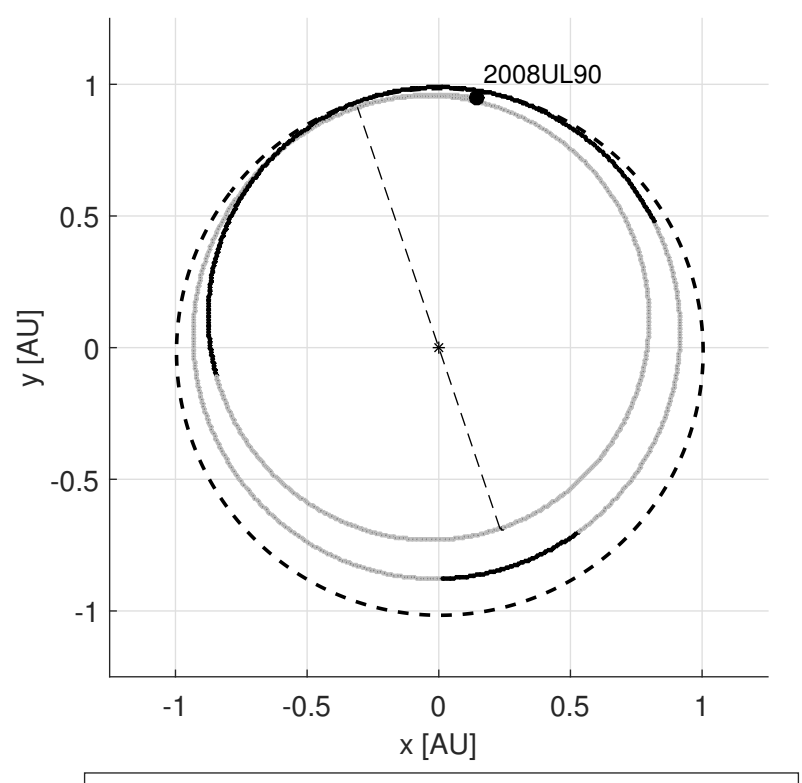

Figure 9: Transfer to parking orbit using electric propulsion.

propellant mass required for a chemical propulsion system (Table 5). The semimajor axis variation and the thrust angle profile during the trajectory are reported in Figure 10, together with a 0/1 flag representing the Off/On conditions of the engine. The fly-bys are indicated by the vertical lines.

A reduction of the $\Delta V$ required to lower the perihelion is theoretically achievable by exploiting multiple shallow swing-by's of the Earth (see strategy 2 in Section 4). However, after the last fly-by the orbit of the spacecraft has a perihelion equal to $0.8755 \mathrm{AU}$ and an aphelion equal to $0.9633 \mathrm{AU}$. In this configuration the maximum variation of perihelion is limited to 0.0012 AU per revolution of the spacecraft, which implies a very slow transfer to the target surveillance orbit. The use of the Earth resonances has, therefore, not been considered further in this study.

The transfer to the surveillance orbit using one swing-by of the Earth, instead, takes 564.91 days. The minimum altitude from the Earth during the swing-by is $535.37 \mathrm{~km}$ and the total $\Delta V$ to achieve the surveillance orbit is $1.27 \mathrm{~km} / \mathrm{s}$, against the $1.79 \mathrm{~km} / \mathrm{s}$ required for a direct low-thrust spiral. The transfer is shown in Figure 11, where thrust arcs are in black and coast arcs in gray. The coast leg before the encounter with the Earth lasts 63.78 

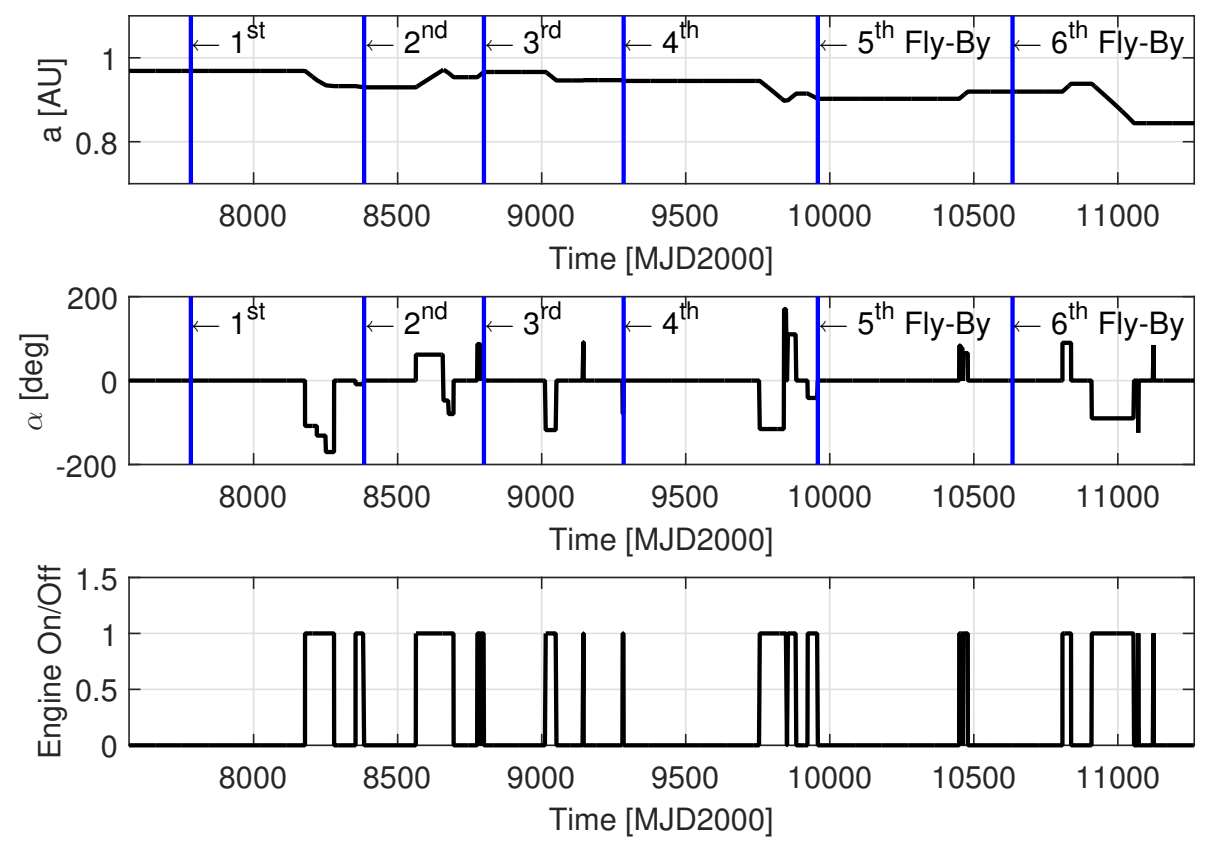

Figure 10: Semi-major axis $(a)$, thrust angle $(\alpha)$, and switching function profiles for lowthrust trajectory. 


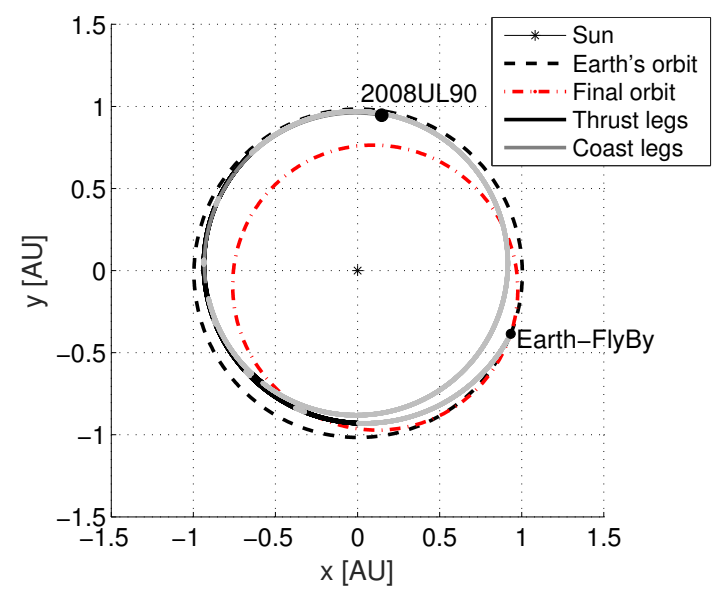

Figure 11: Transfer to parking orbit realised with gravity assist from the Earth.

days. Details of the transfer are given in Table 8.

Table 8: Summary of transfer to surveillance orbit with gravity assist of the Earth.

\begin{tabular}{lllllll}
\hline Departure date & $\begin{array}{l}\text { ToF } \\
\text { [days] }\end{array}$ & $\begin{array}{l}\text { Engine on } \\
\text { [days] }\end{array}$ & $\begin{array}{l}\text { Arrival } \\
\text { date }\end{array}$ & $\begin{array}{l}m_{0} \\
{[\mathrm{~kg}]}\end{array}$ & $\begin{array}{l}m_{f} \\
{[\mathrm{~kg}]}\end{array}$ & $\begin{array}{l}\Delta V \\
{[\mathrm{~km} / \mathrm{s}]}\end{array}$ \\
\hline $2029 / 02 / 11$ & 564.91 & 125.24 & $2030 / 08 / 29$ & 594.17 & 568.41 & 1.27 \\
\hline
\end{tabular}

\subsection{Launch and Orbit Injection}

In this section we demonstrate that the launch and orbit injection strategy proposed in Section 5 can be realised with the Indian Space Research Organisation GSLV-D6 (Geosynchronous Satellite Launch Vehicle) ${ }^{4}$, provided that a restartable upper stage is available. The GSLV-D6 places the spacecraft on a GTO with the following orbital elements :

\footnotetext{
${ }^{4}$ Indian Space Research Organisation - http://www . isro.gov . in/launcher/gslv-d6
} 
Altitude of perigee

Altitude of apogee

Inclination

Argument of perigee

$$
\begin{aligned}
h_{p, G T O} & =170 \mathrm{~km} \\
h_{a, G T O} & =35,975 \mathrm{~km} \\
i_{G T O} & =19 \mathrm{deg} \\
\omega_{G T O} & =178 \mathrm{deg}
\end{aligned}
$$

The heliocentric velocity of the spacecraft at departure from Earth and its geocentric declination are computed with the equations given in Section 5 , resulting in $\left\|\mathbf{v}_{\infty}\right\|=0.65 \mathrm{~km} / \mathrm{s}$ and $\delta=-23.26 \mathrm{deg}$.

If a manoeuvre to inject the spacecraft into the hyperbolic orbit was to be realised at the perigee of the GTO orbit, the following orbital parameters, computed using Eqs. (16) to (18), would be obtained for the hyperbolic orbit: $a_{\text {hyp }}=-9.41 \cdot 10^{5} \mathrm{~km}, e_{\text {hyp }}=1.007$ and $\theta=173.22 \mathrm{deg}$. However, for these values of $\delta$ and $\theta$ and using $\omega_{G T O}=178 \mathrm{deg}$, Eq. (15) yields $\sin i>1$. Since $\delta$ is defined by the geometry of the initial velocity, a change of $i$ and $\omega$ is required to insert the spacecraft into the appropriate hyperbolic orbit, as described in Section 5.

The admissible values of $i_{i n j}$ for the considered values of $\delta$ and for $\omega_{i n j} \in$ $[0,2 \pi]$ are shown in Figure 12a. The $\Delta V$ required for the change of inclination (first manoeuvre) for different values of $i_{i n j}$ is shown in Figure 12b while the $\Delta V$ for the injection into the hyperbolic orbit is shown in Figure 13a. Finally, the total $\Delta V$ is shown in Figure 13b.

The minimum of $\Delta V_{\text {total }}$ is found for $\omega_{i n j}=158 \mathrm{deg}$ and $i_{i n j}=54.98 \mathrm{deg}$. The inclination change requires $\Delta V=0.99 \mathrm{~km} / \mathrm{s}$. The perigee variation and injection into hyperbolic orbit requires a further $\Delta V=1.74 \mathrm{~km} / \mathrm{s}$, for a total velocity variation equal to $\Delta V=2.73 \mathrm{~km} / \mathrm{s}$.

Considering a mass of the spacecraft of $700 \mathrm{~kg}$ and an upper stage with a dry mass of $300 \mathrm{~kg}$ and an $I_{s p}$ of $400 \mathrm{~s}$, the propellant required to inject the spacecraft into the hyperbolic orbit from the initial GTO orbit is 1004 $\mathrm{kg}$ and, therefore, the total launch mass is:

$$
m_{S / C}+m_{U / S}+m_{\text {fuel }}=(700+300+1004) \mathrm{kg}=2004 \mathrm{~kg} .
$$

It is important to stress that the value of $I_{s p}=400 \mathrm{~s}$ for the specific impulse of the upper stage is different from the value of the specific impulse of the chemical engine defined in Section $7.1\left(I_{s p}=321 \mathrm{~s}\right)$, because it refers to 


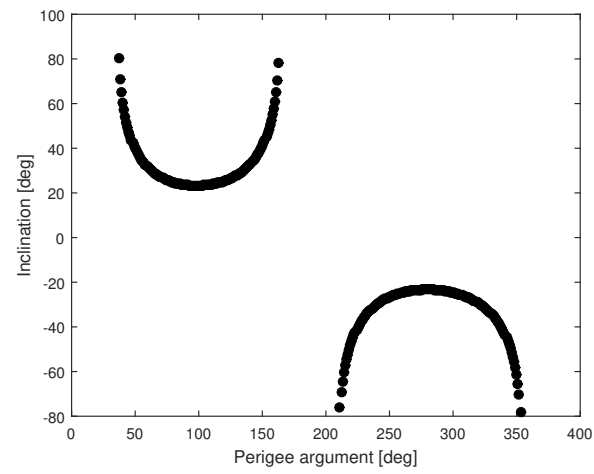

(a)

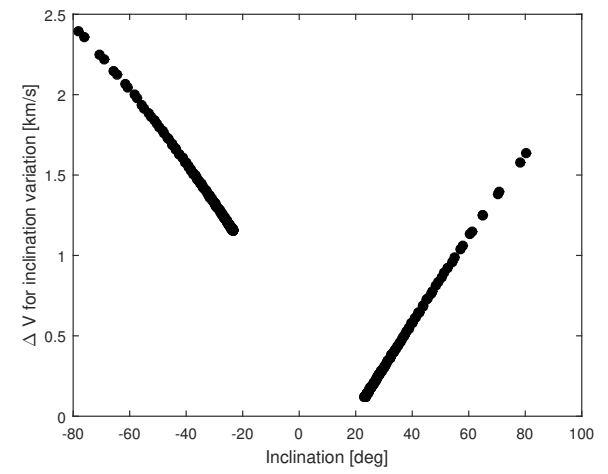

(b)

Figure 12: (a): values of $i_{i n j}$ for different values of $\omega_{i n j}$ allowing injection into the hyperbolic orbit. (b): $\Delta V$ for the variation of inclination from $i_{G T O}$ to $i_{i n j}$ for different values of $i_{\text {inj }}$ (on the x axis).

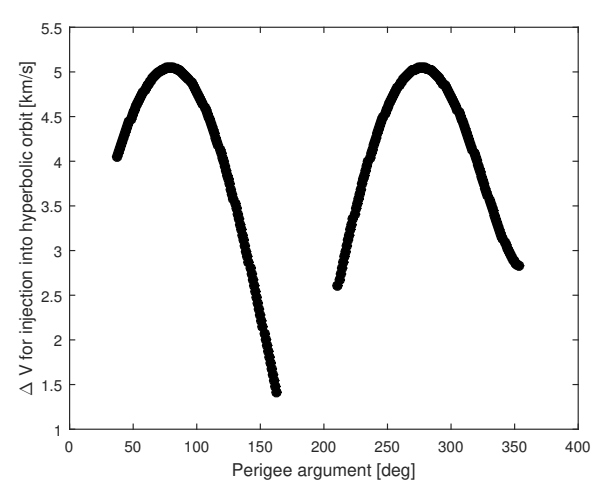

(a)

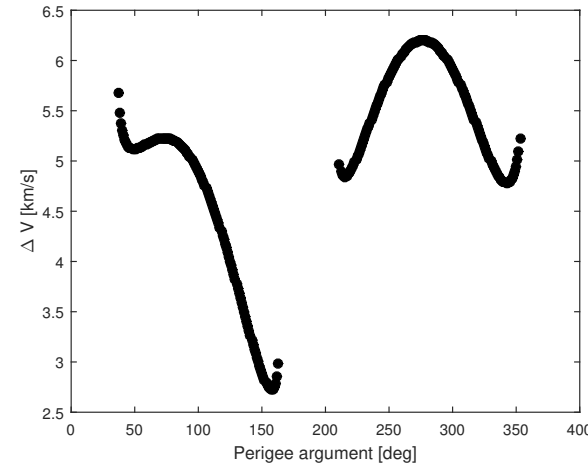

(b)

Figure 13: (a): $\Delta V$ for the injection into the hyperbolic orbit for different values of $\omega_{i n j}$ (on the $\mathrm{x}$ axis). (b): Total $\Delta V$ required to realize the two manoeuvres for the injection into the hyperbolic orbit for different values of $\omega_{i n j}$ (on the x axis). 
a different engine. The dry mass and propellant mass of the upper stage corresponds to a propellant mass fraction equal to 0.77. Based on the launch history and on future planned missions, GSLV-D6 has an upper limit of $2330 \mathrm{~kg}$ in GTO. It would thus accommodate the spacecraft and upper stage leaving a mass margin of $326 \mathrm{~kg}$.

\subsection{Visibility Analysis}

The number and type of asteroids that can be potentially detected over the mission lifetime are estimated by running 100 visibility simulations each with a different synthetic populations of $10^{6}$ IEO, Atens and Apollos. The constraints chosen for the observations are:

- Observations are only carried out when the low-thrust engine is off, at intervals of 5 day.

- The angle $\psi$ (angle Sun-spacecraft-Asteroid) is set to 45 degrees so that the camera does not point directly towards the Sun.

- The declination of the camera is zero degrees as the maximum population of asteroids is close to the ecliptic.

- The limiting relative magnitude of the camera is 19.5 and its FOV is 12 deg.

For the simulations a mean albedo of 0.154 is considered. Figure 14 shows a schematic representation of the geometry of observation. The blue arrow

represents the direction of the instrument, oriented toward the inner part of the orbit; the red arrow is the spacecraft's velocity vector. The dots represent the asteroids detected during the observation.

Figure 15 shows the distribution of objects, in the $a-e$ plane, for the known and synhetic populations of IEOs, Atens and Apollo.

Table 9 shows the results of the 100 simulations for the observations made by the spacecraft over the entire mission time, considering both the synthetic and known NEAs population. The table reports the number of unique Atira, Aten and Apolo asteroids (no repetition of the same asteroids between one observation and the next) observed during the entire mission. Also the diameters of the observed asteroids in each group are presented. Out of the observed NEAs reported in Table 9, 3 Atira, 32 Aten and 108 Apollo belong to the known NEAs population. 


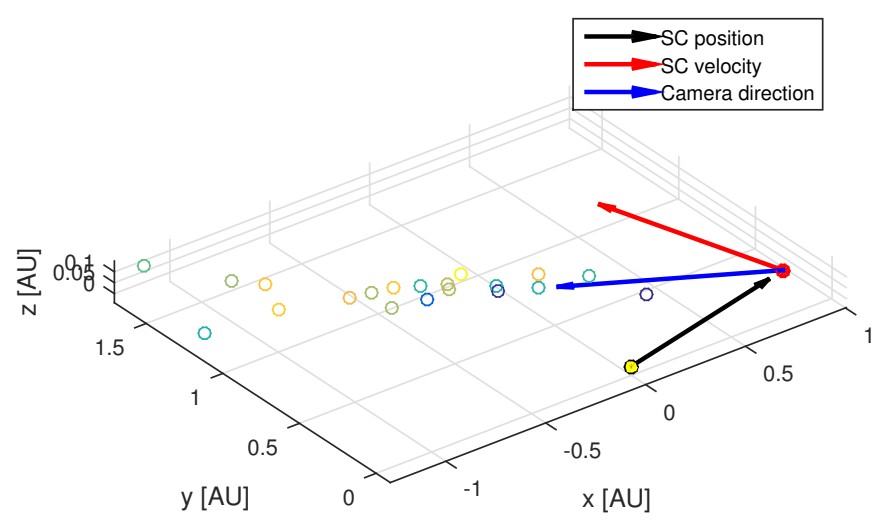

Figure 14: Representation of the asteroids observation.
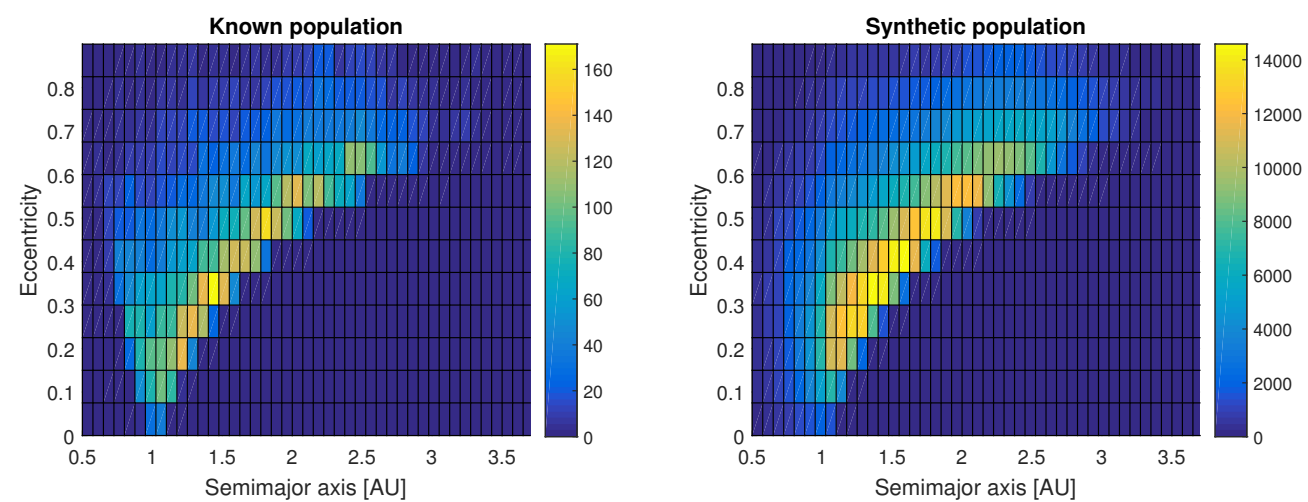

Figure 15: Distribution, in the $a-e$ plane, of the known and synthetic populations of IEOs, Atens and Apollos

The distribution of the diameter of the observed NEAs is shown, for diameter smaller than $10 \mathrm{~km}$, in Figure 16.

Results show that a mean value of 39 IEOs could be detected during the mission lifetime. The mission would also allow to observe, on average, 96 Atens and 364 Apollos. The distribution of the diameter of the observed asteroids of the synthetic populations (Figure 16) shows that most of the observed asteroids have diameter lower than $2 \mathrm{~km}$. In particular, 32 objects with diameter lower than $0.5 \mathrm{~km}$ can be observed. 
Table 9: Results of the visibility analysis using the synthetic and known populations of NEAs.

\begin{tabular}{llll}
\hline & Min & Mean & Max \\
\hline Number observed Atira & 26 & 39 & 60 \\
Number observed Aten & 77 & 96 & 120 \\
Number observed Apollo & 329 & 364 & 405 \\
\hline & Min & Mean & Max \\
\hline Diameter observed Atira $[\mathrm{km}]$ & 0.0989 & 0.9864 & 8.1168 \\
Diameter observed Aten $[\mathrm{km}]$ & 0.0814 & 1.1568 & 8.4291 \\
Diameter observed Apollo $[\mathrm{km}]$ & 0.0637 & 1.5946 & 8.5027 \\
\hline
\end{tabular}

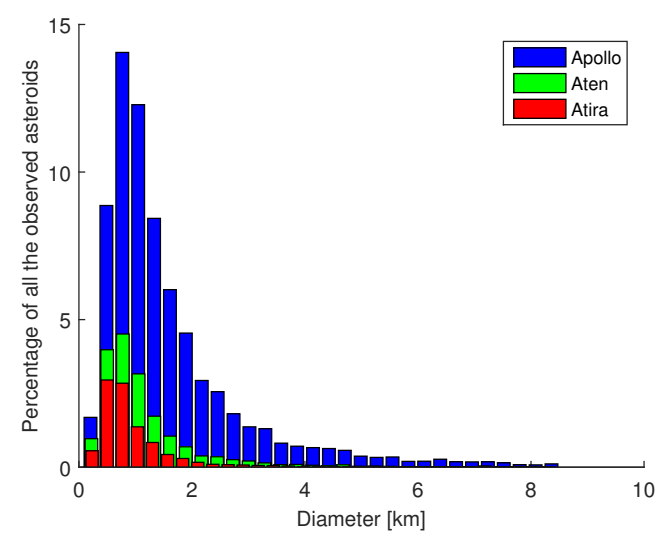

Figure 16: Distribution of the diameter of the observed objects for diameter between 0 and $10 \mathrm{~km}$.

\section{Conclusions}

This paper proposes a multiple-asteroid tour to visit the Atira asteroids using low-thrust propulsion while conducting observations of the inner region of the Solar System in order to possibly detect new NEAs. Hundreds of sequence of asteroid were found by a deterministic branch and prune procedure considering impulsive transfers for different departure and encounter dates and assuming fly-by of the asteroids at their nodal points. The best solution found with this simple procedure was first refined using an evolutionary global search algorithm and then translated into an optimised low-thrust transfer. It has to be noted that the approach proposed in this paper does 
not provide an optimal solution in the optimal control sense but a feasible solution with a conservative estimation of the propellant cost. A further improvement is, therefore, expected by relaxing some of the terminal constraints and solving a more accurate optimal control problem. Results show that six asteroids of the Atira group can be visited through fly-by's over a period of approximately 8.4 years, with a small compact $700 \mathrm{~kg}$ spacecraft launched by a GSLV-D6.

The paper demonstrated that the tour of the Atira asteroids offers two additional opportunities. After the last asteroid the spacecraft can be placed, with a swing-by of the Earth, on a surveillance orbit with perihelion at Venus. This orbit offers a vantage point to observe and monitor the asteroids in the inner Solar System.

Furthermore, during the whole mission lifetime an asteroid survey campaign, using a small and compact instrument, can potentially discover on average 364 additional Near Earth Asteroids out of which 39 could belong to the IEO group.

\section{Acknowledgments}

This research was partially founded by Airbus Defence and Space and by the Marie Curie FP7-PEOPLE-2012-ITN Stardust. The authors would like to thank Mr Stephen Kemble for his support and contribution.

\section{References}

Becerra, V.M., Myatt, D.R., Nasuto, S.J., Bishop, J.M. \& Izzo, D., An efficient pruning technique for the global optimisation of multiple gravity assist trajectories, Acta Futura, Vol. 1, pp. 35-42, 2005.

Bonnard, B., Faubourg, L. \& Trélat, E., Mećanique céleste et contrôle des véhicules spatiaux, Springer Science \& Business Media, 2005.

Brophy, J.R., Rayman, M.D., \& Pavri, B., Dawn: An ion-propelled journey to the beginning of the solar system, 2008 IEEE Aerospace Conference, Big Sky, MT, pp. 1-10, 2008. doi: 10.1109/AERO.2008.4526264

Chobotov, V.A., Orbital mechanics, AIAA Education Series, 2002. 
Greenstreet, S., Ngo, H., \& Gladman, B., The orbital distribution of NearEarth Objects inside Earth's orbit, Icarus, Vol. 217, pp. 355-366, 2012. doi: $10.1016 / j$. icarus. 2011.11.010

Harris, A., NEA populations and impact frequency, NASA Asteroid Grand Challenge Seminar, 2014. http://sservi .nasa.gov/wp-content/ uploads/2014/03/Harris.pdf

Kawaguchi, J.I., The Hayabusa mission - Its seven years flight, 2011 IEEE Symposium on VLSI Circuits, Honolulu, HI, pp. 2-5, 2011.

Kemble, S., Interplanetary mission analysis and design, Springer Science \& Business Media, 2006.

Minisci, E. \& Vasile, M., Adaptive Inflationary Differential Evolution, 2014 IEEE Congress on Evolutionary Computation, Beijing, pp. 1792-1799, 2014. doi: 10.1109/CEC.2015.7256950

Novak, D. \& Vasile, M., Incremental solution of LTMGA transfers transcribed with an advanced shaping approach, 61st International Astronautical Congress, Prague, 2010.

Price, K., Storn, R.M. \& Lampinen, J.A., Differential evolution: a practical approach to global optimization, Springer Science and Business Media, 2006. doi: $10.1007 / 3-540-31306-0$.

Rayman, M.D., \& Williams, S.N., Design of the first interplanetary solar electric propulsion mission, Journal of Spacecraft and Rockets, Vol. 39, Issue 4, pp. 589-595, 2002.

Sauer, C.G. \& Yen, C.W.L., Planetary mission capability of small low power Solar Electric Propulsion systems, Acta Astronautica, Vol. 35, Supplement 1, pp. 625-634, 1995. doi: doi :10.1016/0094-5765 (94)00230-J

Sorensen, K., Hyperbolic injection issues for MXER tethers, 39th AIAA/ASME/SA/ASEE Joint Propulsion Conference, Huntsville, AL, 2003.

Stock, A., Investigation on Low Cost Transfer Options to the Earth-Moon Libration Point Region, Diploma Thesis, University of Stuttgart, 2009. https://forum.nasaspaceflight.com/index.php? action=dlattach; topic $=29106$. 0 ; attach $=416980$. 
Stuart, J.S., Observational constraints on the number, albedos, sizes, and impact hazards of the near-Earth asteroids, Doctoral dissertation, Massachusetts Institute of Technology, 2003. https://www.11.mit.edu/ mission/space/linear/files/StuartThesis.pdf

Tedesco, E.F., IRAS minor planet survey, Springer, Netherlands, 1994.

Vasile, M. \& Pascale, P.D., Preliminary design of multiple gravity-assist trajectories, Journal of Spacecraft and Rockets, Vol. 43, Issue 4, pp.794-805, 2006. doi: $10.2514 / 1.17413$

Veres, P. et al., Absolute magnitudes and slope parameters for 250,000 asteroids observed by Pan-STARRS PS1 - preliminary results, Icarus, Vol. 261, pp. 34-47, 2015. doi: 10.1016/j . icarus.2015.08.007

Wallace, B., Scott, R. \& Sale, M., The Near Earth Object Surveillance Satellite: Mission status and CCD evolution after 18 months on-orbit, Advanced Maui Optical and Space Surveillance Technologies Conference, Maui, Hawaii, 2014.

Wales, D.J. \& Doye, J.P., Global optimization by basin-hopping and the lowest energy structures of Lennard-Jones clusters containing up to 110 atoms, The Journal of Physical Chemistry A, 101(28), pp. 5111-5116, 1997. doi: $10.1021 /$ jp970984n

Williams, S.N. \& Coverstone-Carroll, V., Benefits of solar electric propulsion for the next generation of planetary exploration missions, Journal of the Astronautical Sciences, Vol. 45, Issue 2, pp. 143-159, 1997.

Zuiani, F., Vasile, M., Palmas, A. \& Avanzini, G., Direct transcription of low-thrust trajectories with finite trajectory elements, Acta Astronautica, Vol. 72, pp. 108-120, 2012. doi: 10.1016/j .actaastro.2011.09.011

Zuiani, F. \& Vasile, M., Extended analytical formulas for the perturbed Keplerian motion under a constant control acceleration, Celestial Mechanics and Dynamical Astronomy, Vol. 121, Issue 3, pp. 275-300, 2015. doi: $10.1007 / \mathrm{s} 10569-014-9600-5$ 